\title{
Falls in Parkinson's Disease Subtypes: Risk Factors, Locations and Circumstances
}

\author{
Paulo H. S. Pelicioni ${ }^{1}$, Jasmine C. Menant ${ }^{1}$, Mark D. Latt ${ }^{2}$ and Stephen R. Lord ${ }^{1, *}$ \\ 1 Neuroscience Research Australia, School of Public Health and Community Medicine, \\ University of New South Wales, Sydney, NSW 2052, Australia; p.pelicioni@neura.edu.au (P.H.S.P.); \\ j.menant@neura.edu.au (J.C.M.) \\ 2 Department of Aged Care, Royal Prince Alfred Hospital, Sydney, NSW 2050, Australia; \\ mark.latt@sydney.edu.au \\ * Correspondence: s.lord@neura.edu.au; Tel.: +61-2-9399-1061
}

Received: 22 May 2019; Accepted: 19 June 2019; Published: 23 June 2019

\begin{abstract}
People with Parkinson's disease (PD) can be classified into those with postural instability and gait difficulty (PIGD subtype) and those manifesting tremor as the main symptoms (non-PIGD subtype). In a prospective cohort study of 113 people with PD we aimed to contrast fall rates and circumstances as well as a range of disease-related, clinical, and functional measures between the PD subtypes. Compared with non-PIGD participants, PIGD participants were significantly more likely to suffer more falls overall as well as more falls due to freezing of gait, balance-related falls and falls at home. The PIGD group also performed significantly worse in a range of fall-related clinical and functional measures including general cognitive status, executive function, quadriceps muscle strength, postural sway and the timed up and go test. These findings document the extent to which people with the PIGD subtype are at increased risk of falls, the circumstances in which they fall and their disease-related, clinical and functional impairments.
\end{abstract}

Keywords: Parkinson's disease; accidental falls; subtypes; postural balance; executive function; freezing of gait

\section{Introduction}

Falls are a significant cause of disability, lost independence and reduced quality of life in people with Parkinson's disease (PD) [1,2]. Prospective studies show that between $45 \%$ and $68 \%$ of people with PD will fall each year [3,4], with a large proportion (50-86\%) falling recurrently [5]. In addition, their risk of falls and fractures rise steadily from 40 years of age, much earlier than in healthy individuals [6]. The consequences of falls are devastating and include restriction of activities of daily living, fear of falling, high levels of caregiver stress and injuries [1]. In fact, the incidence of hip fracture is four times that for older people of the same age without PD [7]. This has significant economic consequences as the costs of fall-related fractures in people with PD are close to double those in healthy older people [2].

Many risk factors for falls in PD have been identified. These include freezing of gait (FOG), cognitive impairment, poor leaning balance, previous falls, lower limb weakness and slow gait speed $[4,8,9]$. In addition, people with PD with the postural instability and gait difficulty (PIGD) subtype have also been identified as having an increased risk of falls. This subtype has a predominance of postural instability and gait impairment as opposed to the tremor dominant (TD) subtype, for which there is a predominance of resting and postural tremor [10]. Only a few studies, however, have investigated whether people with the PIGD subtype fall more frequently [11-13], and such work is based solely on retrospective surveys, limited by differential to recall bias. 
The circumstances in which falls occur may provide insights into underlying causes of falls and possible fall prevention strategies. For example, in people with PD, falls that occur outdoors are primarily due to slips and trips, whereas falls that occur indoors are related more strongly to lower limb weakness, vertigo and postural instability [14]. However, no studies to date have examined differences between the PIGD and TD subtypes in the circumstances of falls, including fall locations. In addition, only a few studies have contrasted cognitive, functional and mobility measures between the two PD subtypes $[10,11,15]$. Such between-group comparisons are important to identify key impairments that might be amenable to intervention and hence guide the prescription of evidence-based treatments in clinical practice.

Therefore, the aims of this study were to determine differences between the PIGD and non-PIGD PD subtypes in: (1) incidence of falls in a prospective study design, (2) fall locations and fall types, relating to the occurrence of FOG, balance impairment and syncope and (3) a range of disease-related, cognitive, functional and mobility measures. We hypothesised that compared with the non-PIGD group, the PIGD subtype would suffer more falls due to syncope, instability and FOG; more falls at home and more falls overall; and exhibit cognitive, functional and mobility impairments on clinical assessment.

\section{Materials and Methods}

\subsection{Participants}

This study comprised a secondary analysis from a prospective study of falls [8]. One hundred and thirteen people with idiopathic PD diagnosed according to the UK PD Brain Bank criteria [16] were recruited from within the city of Sydney, NSW. Volunteers were recruited from the following sources: $36(31 \%)$ from a hospital outpatient clinic, $15(13 \%)$ from a volunteer database, 20 (18\%) from PD support groups and $42(37 \%)$ from the general community. The exclusion criteria comprised: An inability to walk unassisted without a walking aid during the tests, a Mini-Mental State Examination (MMSE) [17] score <24, an atypical Parkinsonian syndrome evidence of psychosis, neuroleptic use, vertigo, epilepsy, stroke, transient ischaemic attacks, syncope, uncompensated heart failure or valvular heart disease. Participants were assessed in their typical "on" phase of the levodopa treatment cycle. The protocol was approved by the Human Studies Ethics Committee at the University of Sydney (approval number: HREC 2002/3/4.18 (1401)) and informed consent was obtained from all participants prior to their participation.

\subsection{Assessments}

\subsubsection{Subtype Classification}

The mean tremor score was calculated as the mean of Unified Parkinson's Disease Rating Scale (UPDRS) part II, item 16 (tremor) and UPDRS part III, items 20 (rest tremor) and 21 (action tremor) scores. The mean PIGD score was calculated as the mean of UPDRS part II, items 13 (falling), 14 (freezing) and 15 (walking) and UPDRS III, items 29 (gait) and 30 (postural stability) scores [10]. The ratio of mean tremor score to mean PIGD score was then calculated to determine the PD subtype: ratios $\geq 1.5$ identified participants with the TD subtype $(n=13)$, ratio scores $\leq 1.0$ the PIGD subtype $(n=67)$ and ratios between 1.01 and 1.49 the indeterminate subtype $(n=2)$. Due to relatively small sample numbers, the TD and indeterminate groups were combined to form a single non-PIGD group $(n=46)$ for all analyses [10].

\subsubsection{PD-Related and Health Measures}

In addition to the above PD subtype classification, several other PD related measures were collected. These included duration of disease since first symptoms; stage of the disease according to the Hoehn and Yahr scale [18]; Presence of Rigidity, Axial Posture, Bradykinesia and Dyskinesia according to the UPDRS items; UPDRS part I, II, II, IV and total scores [19]; levodopa daily dosage, 
dopamine agonist, anticholinergic medication and Catechol-O-Methyl Transferase (COMT) inhibitor use. In addition, information on falls in the past year, walking aid use inside and outside the home and non-PD medication use was recorded.

\subsubsection{Cognition}

The MMSE was used to assess global cognition [17] and the Frontal Assessment Battery (FAB) was used to assess executive functioning [20].

\subsubsection{Sensorimotor, Balance, Gait and Mobility}

Participants were assessed using the Physiological Profile Assessment (PPA) [21] which comprises tests evaluating key functions of the human balance system: Peripheral sensation, visual contrast sensitivity, lower limb strength, simple reaction time and postural sway when standing on a compliant surface. An individual composite fall risk score was computed from an algorithm including the scores achieved in each test. This physiological fall risk assessment has been shown to predict recurrent falls in community-dwelling older people with an accuracy of 75\% [22]. The coordinated stability test was used to assess controlled leaning balance, i.e. how participants adjust their balance in a steady and coordinated manner when near the limits of their base of support. Higher scores indicate poorer dynamic postural stability [23].

Gait analysis was performed using a tri-axial piezo-resistant accelerometer attached to the participant's pelvis on a belt at the level of the sacrum. Participants performed one walking trial at self-selected speed along a $20 \mathrm{~m}$ corridor and data collected from the middle $15 \mathrm{~m}$ of steady state walking were analysed. Average gait speed was calculated by dividing the walking distance by the total time taken to complete the distance. Step time variability was computed from the extracted acceleration data (standard deviations between successive heel contacts over the middle $15 \mathrm{~m}$ of steady state walking) [15]. Step time variability was calculated from the average of 32 steps (range 14-204).

Functional mobility was assessed with the timed up and go (TUG) test [24]. Participants were asked to rise from a chair, walk forward three meters at their usual walking pace, turn 180 degrees, walk back to the chair and sit down.

\subsubsection{Orthostatic Hypotension}

Orthostatic hypotension was defined as a fall in systolic blood pressure by $20 \mathrm{mmHg}$ or more and/or in diastolic blood pressure by $10 \mathrm{mmHg}$ or more, recorded with a sphygmomanometer on the left arm, during the first 3 min of standing up from sitting [25].

\subsubsection{Falls}

Falls were defined as unexpected events which resulted in the participant unintentionally coming to the ground, floor or other lower level [26]. Falls were collected prospectively for 12 months using monthly calendars. All participants who reported a fall were telephoned by a single experienced geriatrician (M.D.L.) to verify the falls circumstances and any related injuries [26]. We classified falls within three types: falls that occurred immediately following FOG (FOG falls); Falls resulting from a slip, trip or loss of balance (balance-related falls) [27]; and falls due to possible syncope or pre-syncope (syncopal and other falls) [27]. Falls that occurred inside the participant's house or immediate surroundings (outside stairs, garage, and garden) were classified as at home falls, with the remainder classified as away from home falls.

\subsection{Statistical Analysis}

Continuous data were inspected for skewed distributions, and log-transformed if required. Differences between PIGD and non-PIGD groups with respect to demographics, physical, disease-related, clinical and functional variables were examined with Student $t$-tests for independent 
samples (normally distributed data), Mann-Whitney $U$-tests (non-parametric data) or chi square tests (categorical data). These data were also compared between the PIGD and non-PIGD groups adjusting for disease duration: analysis of covariance (continuous measures) or Mantel-Haenzel adjustments (categorical data). For the Mantel-Haenzel adjustments, disease duration was categorized as short ( $\leq 5$ years, $n=33.6 \%$ ), medium ( $6-11, n=35.4 \%$ ) and long durations ( $\geq 12$ years, $n=31 \%$ ). Finally, the relationship between PD subtype groups and fall outcomes were examined using the incidence rate ratios from negative binomial regression adjusting by disease duration. Geometric means of fall rates for the two groups are also presented. Geometric means provide a measure of central tendency that uses the product of the values of a variable (as opposed to the arithmetic mean which uses their sum) to accommodate for right-skewed distributions [28]. For these calculations, 0.5 was added to numerators so data for participants with no falls during the 12-months follow-up could be included. Data were analysed using IBM SPSS v. 25 for Windows (SPSS, Inc., Chicago, IL) and significance levels were set at 0.05 .

\section{Results}

\subsection{Parkinson's Disease and Health-Related Characteristics of the PIGD and Non-PIGD Participants}

Table 1 presents the demographic, disease-related and clinical characteristics data of the PIGD and non-PIGD participants. No between-group differences were found for age, sex, height or body mass. In unadjusted analyses, the participants with PIGD had longer disease durations, higher UPDRS parts I, II, IV and total scores, and more likely to have more advanced Hoehn and Yahr stages, worse leg/axial rigidity, worse dyskinesia, FOG and higher levodopa dosage intake. Participants with PIGD were also more likely to be taking anticholinergic medications and report previous falls and the use of walking aids inside and outside of their homes. With the exception of UPDRS part IV scores, dyskinesia and anticholinergic medication use, these associations remained statistically significant when adjusting for Parkinson's disease duration.

Table 1. Parkinson's disease and health-related characteristics for the non-postural instability and gait difficulty (PIGD) and PIGD groups. Data are presented as mean (SD) unless stated otherwise.

\begin{tabular}{|c|c|c|c|c|}
\hline & $\begin{array}{c}\text { Non-PIGD } \\
(n=46)\end{array}$ & $\begin{array}{l}\text { PIGD } \\
(n=67)\end{array}$ & $\begin{array}{c}\text { Unadjusted } \\
\qquad p\end{array}$ & $\begin{array}{c}\text { Adjusted \# } \\
p\end{array}$ \\
\hline \multicolumn{5}{|l|}{ Demographic } \\
\hline Sex (\% Men $)$ & $26(56)$ & $38(56)$ & 0.984 & - \\
\hline Age (years) & $66.3(9.7)$ & $66.1(9.5)$ & 0.917 & - \\
\hline Height (cm) & $171(8)$ & $171(9)$ & 0.781 & - \\
\hline Body Mass (kg) & $72(12)$ & $73(19)$ & 0.938 & - \\
\hline \multicolumn{5}{|l|}{ PD-related } \\
\hline Disease duration (years) & $7.2(6.3)$ & $10.3(6.1)$ & 0.024 & - \\
\hline UPDRS part I (score) & $1.5(1.5)$ & $2.7(1.8)$ & $<0.001$ & 0.008 \\
\hline UPDRS part II (score) & $5.2(3.6)$ & $11.3(6.2)$ & $<0.001$ & $<0.001$ \\
\hline UPDRS part III (score) & $14.9(9.8)$ & $17.1(9.4)$ & 0.161 & 0.28 \\
\hline UPDRS part IV (score) & $2.8(2.6)$ & $3.8(2.8)$ & 0.035 & 0.291 \\
\hline UPDRS total (score) & $26.0(13.8)$ & $36.7(15.5)$ & $<0.001$ & 0.001 \\
\hline TD (score) & $2.8(2.0)$ & $0.7(1.0)$ & $<0.001$ & $<0.001$ \\
\hline PIGD (score) & $1.1(1.1)$ & $4.7(3.0)$ & $<0.001$ & $<0.001$ \\
\hline \multicolumn{5}{|l|}{ Hoehn \& Yahr stages } \\
\hline Stage I $(\%)$ & $25(54)$ & $14(21)$ & & \\
\hline Stage II (\%) & $14(30)$ & $19(28)$ & $<0.001$ & $<0.001$ \\
\hline Stage III (\%) & $6(13)$ & $27(40)$ & & \\
\hline Stage IV (\%) & $1(2)$ & $7(10)$ & & \\
\hline Rigidity (\%)^ & $40(87)$ & $41(61)$ & 0.003 & 0.012 \\
\hline Stooped Posture (\%) & $29(63)$ & $53(79)$ & 0.085 & 0.094 \\
\hline Bradykinesia (\%) & $36(78)$ & $57(85)$ & 0.453 & 0.653 \\
\hline Dyskinesia (\%)^ & $8(17)$ & $24(36)$ & 0.036 & 0.375 \\
\hline
\end{tabular}


Table 1. Cont.

\begin{tabular}{ccccc}
\hline & $\begin{array}{c}\text { Non-PIGD } \\
(\boldsymbol{n}=\mathbf{4 6})\end{array}$ & $\begin{array}{c}\text { PIGD } \\
(\boldsymbol{n}=\mathbf{6 7 )}\end{array}$ & $\begin{array}{c}\text { Unadjusted } \\
\boldsymbol{p}\end{array}$ & $\begin{array}{c}\text { Adjusted \# } \\
\boldsymbol{p}\end{array}$ \\
\hline Levodopa dosage daily intake (\%) & & & & \\
$<750 \mathrm{mg}$ & $40(87)$ & $35(52)$ & $<0.001$ & 0.003 \\
$>750 \mathrm{mg}$ & $6(13)$ & $32(48)$ & $<0.001$ & $<0.001$ \\
FOG (\%) & $8(17)$ & $41(61)$ & 0.020 & 0.105 \\
Anticholinergic medication (\%) & 0 & $8(12)$ & 0.611 & 0.698 \\
Dopamine agonist medication (\%) & $9(20)$ & $10(15)$ & 0.234 & 0.422 \\
COMT inhibitor medication (\%) & $3(6)$ & $10(15)$ & & \\
General health-related & & & $<0.001$ & 0.001 \\
Past fallers (\%) & $15(33)$ & $46(69)$ & 0.017 \\
Walking aid used-inside (\%) & $3(6)$ & $21(31)$ & 0.002 & 0.006 \\
Walking aid used-outside (\%) & $5(11)$ & $27(40)$ & 0.001 & 0.473 \\
Drugs daily intake >5 (\%) & $16(35)$ & $33(49)$ & 0.176 & \\
\hline
\end{tabular}

\# Adjusted for disease duration. PD-Parkinson's disease; UPDRS-Unified Parkinson's Disease Rating Scale; TD: tremor dominant; PIGD: Postural Instability and Gait Difficulty; FOG-Freezing of gait; COMT: Catechol-O-Methyl Transferase. ^ noted as part of the UPDRS assessment.

\subsection{Cognitive, Sensorimotor, Balance, Gait, Mobility and Cardiovascular Measures: PD Subtype Comparisons}

Table 2 presents the cognitive, sensorimotor, balance, gait, mobility and cardiovascular measures for the PIGD and non-PIGD participants. In unadjusted analyses, the participants with PIGD showed lower MMSE and FAB scores, reduced quadriceps strength, greater postural sway, higher PPA scores, worse coordinated leaning balance as assessed with the coordinated stability test, and longer TUG times. With the exception of the PPA and coordinated leaning balance measures, these associations remained statistically significant when adjusting for PD duration.

Table 2. Cognitive, sensorimotor, balance, gait, mobility and cardiovascular characteristics for the non-PIGD and PIGD groups. Data are presented as mean (SD) unless stated otherwise.

\begin{tabular}{ccccc}
\hline & Non-PIGD & PIGD & Unadjusted & Adjusted \# \\
& $(\boldsymbol{n}=\mathbf{4 6 )}$ & $(\boldsymbol{n}=\mathbf{6 7 )}$ & $\boldsymbol{p}$ & $\boldsymbol{p}$ \\
\hline MMSE (score) & $29.6(1.0)$ & $28.7(2.5)$ & 0.004 & 0.046 \\
FAB (score) & $17.1(1.7)$ & $15.1(3.7)$ & 0.001 & 0.002 \\
Contrast Sensitivity (dB) & $19.6(2.1)$ & $19.8(1.8)$ & 0.695 & 0.459 \\
Proprioception (degrees of error) & $2.3(1.1)$ & $2.5(1.5)$ & 0.424 & 0.455 \\
Quadriceps strength (kg) & $24.7(10.7)$ & $20.2(8.6)$ & 0.014 & 0.016 \\
Hand reaction time (ms) & $281(73)$ & $300(109)$ & 0.265 & 0.475 \\
Postural sway (mm) & $159(149)$ & $265(237)$ & 0.008 & 0.047 \\
PPA (score) & $0.95(1.29)$ & $1.58(1.54)$ & 0.021 & 0.156 \\
Coordinated stability (score) & $12.9(14.1)$ & $18.2(11.8)$ & 0.039 & 0.209 \\
Step velocity (m/s) & $1.05(0.19)$ & $0.95(0.34)$ & 0.097 & 0.214 \\
Step time variability (ms) & $67(29)$ & $79(65)$ & 0.491 & 0.611 \\
TUG (s) & $8.3(2.6)$ & $12.5(8.4)$ & $<0.001$ & $<0.001$ \\
Orthostatic hypotension (\%) & $6(13)$ & $10(15)$ & 1 & 0.922 \\
\hline
\end{tabular}

\# Adjusted for disease duration. MMSE-Mini-Mental State Examination; FAB-Frontal Assessment Battery; PPA-Physiological Profile Assessment; TUG-Timed up and go test. Note: higher scores indicate better performances in the MMSE, FAB, contrast sensitivity, quadriceps strength, step velocity and worse performances in the proprioception, hand reaction time, postural sway, PPA, coordinated stability, step time variability and TUG.

\subsection{Falls}

A total of 2043 falls were reported. Of these, 124 falls (6\%) were reported by the non-PIGD and 1919 (94\%) were reported by the PIGD group. In terms of fall types, 1249 were FOG related, 537 were balance related and 257 were syncopal and other falls. Falls related outcome adjusted for disease duration for the non-PIGD and PIGD groups are presented in Table 3. Compared with the 
non-PIGD participants, more PIGD participants suffered falls in general as well as more FOG-related, balance-related and at-home falls. These findings were also mirrored in the fall rate analyses.

Table 3. Fall outcomes in the PD subtypes.

\begin{tabular}{|c|c|c|c|c|}
\hline & & $\begin{array}{c}\text { Non-PIGD } \\
(n=46)\end{array}$ & $\begin{array}{c}\text { PIGD } \\
(n=67)\end{array}$ & Subtype Comparison \\
\hline \multirow{7}{*}{ Fallers } & & $n(\%)$ & $n(\%)$ & IRR (95\% confidence interval) \\
\hline & $\geq 1$ fall & $9(20)$ & $37(55)$ & $3.04(1.46-6.34)$ \\
\hline & $\geq 1$ FOG-related fall & $2(4)$ & $23(34)$ & $6.76(1.58-28.91)$ \\
\hline & $\geq 1$ balance-related fall & $7(15)$ & $26(39)$ & $2.50(1.07-5.86)$ \\
\hline & $\geq 1$ syncopal and other fall & $1(2)$ & $7(10)$ & $5.03(0.60-42.21)$ \\
\hline & $\geq 1$ at home falls $\#$ & $5(11)$ & $30(45)$ & $4.10(1.41-11.91)$ \\
\hline & $\geq 1$ away from home falls \# & $4(9)$ & $10(15)$ & $3.25(1.08-9.76)$ \\
\hline \multirow{7}{*}{ Falls } & & GM $\ddagger$ & GM $\ddagger$ & IRR (95\% confidence interval) \\
\hline & All fall types & 0.83 & 3.48 & $10.21(3.22-32.44)$ \\
\hline & FOG related falls & 0.54 & 1.62 & $156.30(23.89-1022.45)$ \\
\hline & Balance related falls & 0.74 & 1.30 & $4.72(1.33-16.70)$ \\
\hline & Syncopal and other falls & 0.55 & 0.67 & $1.58(0.14-17.41)$ \\
\hline & At home falls $\#$ & 0.66 & 1.76 & $9.94(2.50-39.61)$ \\
\hline & Away from home falls \# & 0.62 & 0.86 & $1.32(0.98-17.87)$ \\
\hline
\end{tabular}

FOG—Freezing of gait; IRR: Incidence Rate Ratio. ${ }^{\#}$ Fall location data from 1014 falls. ${ }^{\ddagger}$ Geometric mean of individual fall rates with 0.5 added to all numerators to enable the inclusion of participants with zero falls during the follow-up period in the comparisons.

\section{Discussion}

In this prospective cohort study of 113 people with $\mathrm{PD}$, we contrasted fall rates and circumstances as well as a range of disease-related, clinical and functional measures between PD motor subtypes. We found that compared with non-PIGD participants, PIGD participants were significantly more likely to suffer more falls overall as well as more falls due to FOG, balance-related falls and falls at home. After adjusting for disease duration, the PIGD group also performed significantly worse in a range of fall-related clinical and functional measures including general cognitive status, executive function, quadriceps muscle strength, postural sway and TUG mobility. These findings are consistent with our hypotheses and document the extent to which people with PD with the PIGD are at elevated risk of falls.

It has been suggested the TD-PIGD subtype distinction may reflect different stages of PD rather than different disorders [29], and as anticipated, the PIGD group had longer disease durations than the non-PIGD group in our cohort. Accordingly, we adjusted for PD duration in our subtype comparisons and some measures (motor complications assessed by UPDRS part IV, dyskinesia, anticholinergic medication, risk of falls assessed by the PPA and controlled leaning balance) did not remain statistically significant. However, many disease-related, cognitive, sensorimotor, balance, gait and mobility measures did remain significant discriminators of PD subtype after adjustment for disease duration. Therefore, it appears the PD subtype classification, as used in this study, is useful for identifying people with PD at increased fall risk as well as elucidating possible underlying causes of falls.

Previous work addressing fall risk in the PIGD subtypes has used retrospective study designs [11-13]. Retrospective designs are not only limited by recall bias, but also by circular comparisons, in that past falls comprise a component of the PIGD subtype classification. In contrast, our prospective findings are free of these limitations and document the greatly increased risk of falls prospectively. Our findings reveal that compared with the non-PIGD group, the PIGD group have a 170 times greater rate of FOG-related falls, a 5 times greater rate of balance-related falls, a 10 times greater rate of at-home falls and a 10 times greater rate of falls overall. In contrast to FOG-related and balance-related falls, the non-PIGD and PIGD groups did not differ with respect to either syncopal and other falls (often associated with pre-syncope or syncope events [27]) or the presence of orthostatic 
hypotension. This suggests that such cardiovascular related factors do not contribute to the elevated fall risk evident in the PIGD group.

In parallel to higher fall rates, the PIGD group had a greater prevalence of disease-related, cognitive, sensorimotor, balance, gait and mobility factors known to increase fall risk in people with PD $[4,8,30]$. While some of these factors comprise measures of disease severity, many help explain why many PIGD subtype participants fall frequently. Our findings of an elevated prevalence of FOG-related falls in the PIGD group complement previous studies $[8,9,30]$ that have shown FOG is a strong risk factor for falls. Furthermore, the reduced quadriceps strength, poor balance and reduced mobility (slower TUG test performance) exhibited by the PIGD group might explain their increased prevalence of balance-related falls.

Bloem et al. [3] have reported that people with PD fall more at home, while healthy older people fall more outside; the former being due to disease-related mobility impairments and the latter due to greater exposure to unexpected hazards and circumstances. Our study builds on this work by showing that those with the PIGD subtype fall significantly more at home than the non-PIGD subtype. In addition to their multiple cognitive and physical impairments, fall risk in the PIGD group may be exacerbated by walking in the more confined space of the home in situations that can trigger FOG such as gait initiation, short walks and turns [31] and subsequent falls. The more similar fall rates between the PD subtypes for falls away from home may reflect those with impaired balance and mobility spending less time away from their homes with resultant limited exposure to this more hazardous environment. Future studies should measure the number of steps of the amount of physical activity required before a person falls to examine this question further.

Strengths of the study include: the inclusion of a diverse range of putative risk factors and the prospective ascertainment of falls over a 12-month period. We also acknowledge certain limitations. First, we had a sample chosen by convenience and due to the relatively small sample the TD and Intermediate subtypes were combined into one non-PIGD group. Second, we acknowledge that despite using the gold standard method of ascertaining monthly falls data via postal calendars and ensuing telephone calls, a recall bias regarding the circumstances of the falls is possible and could have led to some misclassifications of the fall types reported. Third, fall location information was obtained for approximately only half of the reported falls. This was due to the difficulty in reporting such information for those who suffered very frequent falls. Finally, the single centre nature of the study conducted in a metropolitan area may limit the generalizability of the study, even though participants were community-dwellers who were drawn from a range of sources: Hospital outpatients department and support groups. Future studies could include large samples, as well as participants from multiple sites and geographical areas to allow greater generalizability of the study findings. Larger participant numbers in each PD subtype would also allow the investigation of risk factors for falls within each subtype.

Our findings have important clinical implications in that they document clinical, medical and sensorimotor impairments in people with the PD PIGD subtype, some of which may be amenable to intervention. Future studies could address one or more of these risk factors relating to executive dysfunction, FOG, quadriceps weakness, postural instability and poor mobility in randomized controlled trials. Additionally, given the high prevalence of at home falls in the PIGD group, occupational therapy interventions based on safe mobility training and removal of environmental hazards may help prevent falls in the home setting.

\section{Conclusions}

The study findings document the extent to which people with the PIGD subtype are at increased risk of falls, the circumstances in which they fall and their disease-related, clinical and functional impairments. Compared with non-PIGD participants, PIGD participants were significantly more likely to suffer falls, more falls overall, as well as more falls due to FOG, balance-related falls and falls at home. The PIGD group also performed significantly worse in a range of fall-related clinical and functional 
measures including general cognitive status, executive function, quadriceps muscle strength, postural sway and the TUG test. This information may prove useful for informing cognitive, physical and environmental interventions to prevent falls in this high-risk group.

Author Contributions: Conceptualization, M.D.L. and S.R.L.; methodology, M.D.L. and S.R.L.; formal analysis, M.D.L. and P.H.S.P.; writing-original draft preparation, P.H.S.P.; writing-review and editing, M.D.L., J.C.M. and S.R.L.; visualization, J.C.M. and S.R.L.; supervision, S.R.L. and J.C.M.; project administration, S.R.L.; funding acquisition, P.H.S.P., M.D.L. and S.R.L.

Funding: Paulo Pelicioni is a recipient of a Coordenação de Aperfeiçoamento de Pessoal do Nível Superior (CAPES) PhD scholarship [Grant number: BEX 2194/15-5] and Mark Latt was funded by a University of Sydney $\mathrm{PhD}$ scholarship to undertake this study. Stephen Lord is supported by a NHMRC Research Fellowship.

Conflicts of Interest: S.R.L. declares that the physiological profile assessment (NeuRA FallScreen) is commercially available through neuroscience Research Australia. The other authors declare no conflicts of interest.

\section{References}

1. Fasano, A.; Canning, C.G.; Hausdorff, J.M.; Lord, S.; Rochester, L. Falls in Parkinson's disease: A complex and evolving picture. Mov. Disord. 2017, 32, 1524-1536. [CrossRef] [PubMed]

2. Pressley, J.C.; Louis, E.D.; Tang, M.X.; Cote, L.; Cohen, P.D.; Glied, S.; Mayeux, R. The impact of comorbid disease and injuries on resource use and expenditures in parkinsonism. Neurology 2003, 60, 87-93. [CrossRef] [PubMed]

3. Bloem, B.R.; Grimbergeb, Y.A.M.; Cramer, M.; Willemsen, M.; Zwinderman, A.H. Prospective assessment of falls in Parkinson's disease. J. Neurol. 2001, 248, 950-958. [CrossRef] [PubMed]

4. Paul, S.S.; Sherrington, C.; Canning, C.G.; Fung, V.S.C.; Close, J.C.; Lord, S.R. The relative contribution of physical and cognitive fall risk factors in people with Parkinson's disease: A large prospective cohort study. Neurorehabilit. Neural Repair 2014, 28, 282-290. [CrossRef] [PubMed]

5. Allen, N.E.; Schwarzel, A.K.; Canning, C.G. Recurrent falls in Parkinson's disease: A systematic review. Parkinsons Dis. 2013, 2013, 906274. [CrossRef]

6. Kalilani, L.; Asgharnejad, M.; Palokangas, T.; Durgin, T. Comparing the incidence of falls/fractures in Parkinson's disease patients in the US population. PLoS ONE 2016, 11, e0161689. [CrossRef] [PubMed]

7. Walker, R.W.; Chaplin, A.; Hancock, R.L.; Rutherford, R.; Gray, W.K. Hip fractures in people with idiopathic Parkinson's disease: Incidence and outcomes. Mov. Disord. 2013, 28, 334-340. [CrossRef]

8. Latt, M.D.; Lord, S.R.; Morris, J.G.L.; Fung, V.S.C. Clinical and physiological assessments for elucidating falls risk in Parkinson's disease. Mov. Disord. 2009, 24, 1280-1289. [CrossRef]

9. Pelicioni, P.H.S.; Menant, J.C.; Henderson, E.; Latt, M.D.; Brodie, M.A.; Lord, S.R. Mediating factors for the association between executive dysfunction and falls in people with Parkinson's disease. Parkinsonism Relat. Disord. 2019, submitted.

10. Jankovic, J.; McDermott, M.; Carter, J.; Gauthier, S.; Goetz, C.; Golbe, L.; Huber, S.; Koller, W.; Olanow, C.; Shoulson, I.; et al. Variable expression of Parkinson's disease: A base-line analysis of the DATATOP cohort. Neurology 1990, 40, 1529-1534. [CrossRef]

11. Herman, T.; Weiss, A.; Brozgol, M.; Giladi, N.; Hausdorff, J.M. Gait and balance in Parkinson's disease subtypes: Objective measures and classification considerations. J. Neurol. 2014, 261, 2401-2410. [CrossRef] [PubMed]

12. Hiorth, Y.H.; Lode, K.; Larsen, J.P. Frequencies of falls and associated features at different stages of Parkinson's disease. Eur. J. Neurol. 2013, 20, 160-166. [CrossRef] [PubMed]

13. Matinolli, M.; Korpelainen, J.T.; Korpelainen, R.; Sotaniemi, K.A.; Virranniemi, M.; Myllyla, V.V. Postural sway and falls in Parkinson's disease: A regression approach. Mov. Disord. 2007, 22, 1927-1935. [CrossRef] [PubMed]

14. Gazibara, T.; Pekmezovic, T.; Tepavcevic, D.K.; Tomic, A.; Stankovic, I.; Kostic, V.S.; Svetel, M. Circumstances of falls and fall-related injuries among patients with Parkinson's disease in an outpatient setting. Geriatr. Nurs. 2014, 35, 364-369. [CrossRef] [PubMed]

15. Pelicioni, P.H.S.; Brodie, M.A.; Latt, M.D.; Menant, J.C.; Menz, H.B.; Fung, V.S.C.; Lord, S.R. Head and trunk stability during gait before and after levodopa intake in Parkinson's disease subtypes. Exp. Gerontol. 2018, 111, 78-85. [CrossRef] [PubMed] 
16. Hughes, A.J.; Daniel, S.E.; Kilford, L.; Lees, A.J. Accuracy of clinical diagnosis of idiopathic Parkinson's disease: A clinic-pathological study of 100 cases. J. Neurol. Neurosurg. Psychiatry 1992, 55, 181-184. [CrossRef]

17. Folstein, M.F.; Folstein, S.E.; McHugh, P.R. Mini-mental state. A practical method for grading the cognitive state of patients for the clinician. J. Psychiatr. Res. 1975, 12, 189-198. [CrossRef]

18. Hoehn, M.M.; Yahr, M.D. Parkinsonism: Onset, progression and mortality. Neurology 1967, 17, 427-442. [CrossRef]

19. Fahn, S.; Elton, R.L. Unified Parkinson's Disease Rating Scale. In Recent Developments in Parkinson's Disease, 1st ed.; Fahn, S., Mardsen, C.D., Calne, D., Goldstein, M., Eds.; Macmillan Healthcare Information: Florham Park, NJ, USA, 1987; pp. 154-196.

20. Dubois, B.; Slachevsky, A.; Litvan, I.; Pillon, B. The FAB: A Frontal Assessment Battery at bedside. Neurology 2000, 55, 1621-1626. [CrossRef]

21. Lord, S.R.; Menz, H.B.; Tiedemann, A. A physiological profile approach to falls risk assessment and prevention. Phys. Ther. 2003, 83, 237-252.

22. Lord, S.R.; Ward, J.A.; Williams, P.; Anstey, K. Physiological factors associated with falls in older community-dwelling women. J. Am. Geriatr. Soc. 1994, 42, 1110-1117. [CrossRef] [PubMed]

23. Lord, S.R.; Ward, J.A.; Williams, P. Exercise effect on dynamic stability in older women: A randomized controlled trial. Arch. Phys. Med. Rehabil. 1996, 77, 232-236. [CrossRef]

24. Podsiadlo, R.; Richardson, M.D. The timed up and go: A test of basic functional mobility for frail elderly persons. J. Am. Geriatr. Soc. 1991, 39, 142-148. [CrossRef] [PubMed]

25. Allcock, L.M.; Kenny, R.A.; Burn, D.J. Clinical phenotype of subjects with Parkinson's disease and orthostatic hypotension: Autonomic symptom and demographic comparison. Mov. Disord. 2006, 21, 1851-1855. [CrossRef] [PubMed]

26. Lamb, S.E.; Jorstad-Stein, E.C.; Hauer, K.; Becker, C.; Prevention of Falls Network Europe and Outcomes Consensus Group. Development of a common outcome data set for fall injury prevention trials: The Prevention of Falls Network Europe consensus. J. Am. Geriatr. Soc. 2005, 53, 1618-1622. [CrossRef]

27. Menant, J.C.; Wong, A.K.W.; Trollor, J.N.; Close, J.C.T.; Lord, S.R. Depressive symptoms and orthostatic hypotension and risk factors for unexplained falls in community-living older people. J. Am. Geriatr. Soc. 2016, 64, 1073-1078. [CrossRef] [PubMed]

28. Chivers-Seymour, K.; Pickering, R.; Rochester, L.; Roberts, H.C.; Ballinger, C.; Hulbert, S.; Kunkel, D.; Marian, I.R.; Fitton, C.; McIntosh, E.; et al. Multicentre, randomized controlled trial of PDSAFE, a physiotherapist-delivered fall prevention programme for people with Parkinson's. J. Neurol. Neurosurg. Psychiatry 2019, in press. [CrossRef] [PubMed]

29. Nutt, J.G. Motor subtype in Parkinson's disease: Different disorders or different stages of disease. Mov. Disord. 2016, 31, 957-961. [CrossRef]

30. Paul, S.S.; Canning, C.G.; Sherrington, C.; Lord, S.R.; Close, J.C.T.; Fung, V.S.C. Three simple clinical tests to accurately predict falls in people with Parkinson's disease. Mov. Disord. 2013, 28, 655-662. [CrossRef]

31. Pereira, M.P.; Gobbi, L.T.B.; Almeida, Q.J. Freezing of gait in Parkinson's disease: Evidence of sensory rather than attentional mechanisms through muscle vibration. Parkinsonism Relat. Disord. 2016, 29, 78-82. [CrossRef]

(C) 2019 by the authors. Licensee MDPI, Basel, Switzerland. This article is an open access article distributed under the terms and conditions of the Creative Commons Attribution (CC BY) license (http://creativecommons.org/licenses/by/4.0/). 